Case Report

\title{
The Effectiveness of Hepatic Arterial Infusion Chemotherapy with 5-Fluorouracil/Cisplatin and Systemic Chemotherapy with Ramucirumab in Alpha-Fetoprotein-Producing Gastric Cancer with Multiple Liver Metastases
}

\author{
Yasuhiro Doi ${ }^{D},{ }^{1}$ Yasushi Takii, ${ }^{2}$ Kenji Mitsugi, ${ }^{1}$ Koichi Kimura, ${ }^{3}$ and Yutarou Mihara ${ }^{4}$ \\ ${ }^{1}$ Department of Oncology, Hamanomachi Hospital, Fukuoka, Japan \\ ${ }^{2}$ Department of Internal Medicine, Munakata Medical Association Hospital, Munakata, Japan \\ ${ }^{3}$ Department of Surgery, Munakata Medical Association Hospital, Munakata, Japan \\ ${ }^{4}$ Department of Pathology, Kurume University Hospital, Kurume, Japan \\ Correspondence should be addressed to Yasuhiro Doi; doiyasuhirofield@yahoo.co.jp
}

Received 30 August 2018; Revised 18 October 2018; Accepted 28 October 2018; Published 11 November 2018

Academic Editor: Raffaele Palmirotta

Copyright (C) 2018 Yasuhiro Doi et al. This is an open access article distributed under the Creative Commons Attribution License, which permits unrestricted use, distribution, and reproduction in any medium, provided the original work is properly cited.

\begin{abstract}
Alpha-fetoprotein- (AFP-) producing gastric cancer (AFPGC) is characterized by a high incidence of liver and lymph node metastases and poor prognosis. Although several case reports have described successful multidisciplinary treatment, there are currently no standard therapies for AFPGC. A 57-year-old man presented with upper abdominal pain. His serum AFP level was extremely high $(588.9 \mathrm{ng} / \mathrm{mL})$. Computed tomography (CT) revealed multiple liver metastases with several lesions at an imminent risk of rupture. Five days after admission to our hospital, one lesion ruptured. Transarterial chemoembolization (TACE) of the ruptured tumor was performed, and hepatic arterial infusion chemotherapy (HAIC) with 5-fluorouracil (5-FU)/ cisplatin (CDDP) to the other liver metastases was administered. The patient's AFP levels decreased to $297.1 \mathrm{ng} / \mathrm{mL}$. Gastrointestinal endoscopy revealed Borrmann type 2 lesion in the pyloric portion. Pathological examination indicated hepatoid adenocarcinoma of the stomach and metastatic liver. The final diagnosis was AFPGC and multiple liver metastases. The patient underwent systemic chemotherapy with capecitabine/CDDP (cape/CDDP) for three months. His AFP level increased extremely, and CT revealed progression of the liver metastases. TACE was performed, and HAIC (5FU/CDDP) was administered to the progressive lesion of the liver. Originating from the gastric lesion, a distal gastrectomy and $\mathrm{D} 2+\alpha$ lymph node resection were performed. One month after the operation, the patient underwent systemic chemotherapy with paclitaxel/ramucirumab (PTX/ RAM). After eight cycles of chemotherapy, his AFP level had declined, and CT showed a complete response. After three months of drug withdrawal, the patient has undergone maintenance treatment with RAM. It has been two years since the recurrence. Our experience suggests that HAIC with 5-FU/CDDP and systemic chemotherapy with a regimen including RAM may be an effective treatment for AFPGC.
\end{abstract}

\section{Introduction}

Alpha-fetoprotein- (AFP-) producing gastric cancer (AFPGC) is a relatively rare type of malignancy that comprises about $1.1-8.8 \%$ of all gastric cancers [1-3]. AFPGC is characterized by a high incidence in the liver and lymph nodes and poor prognosis [1-3]. There is no standard therapy for this subtype of gastric cancer. However, several case reports have described successful multidisciplinary therapy.

In this study, we presented a case of AFPGC in which the patient has remained alive for 30 months without recurrence after receiving distal gastrectomy with $\mathrm{D} 2+\alpha$ dissection, 


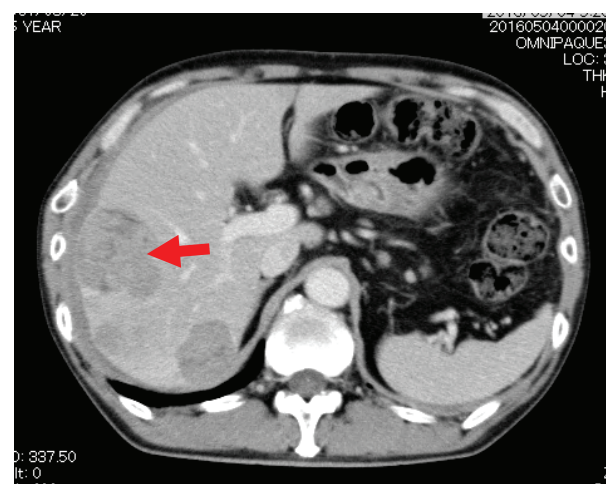

FIGURE 1: CT scan showing multiple liver metastases suggestive of hepatocellular carcinoma. The red arrow indicates the mass rupturing imminently.

hepatic arterial infusion (HAI), and systemic chemotherapy with paclitaxel/ramucirumab (PTX/RAM).

\section{Case Report}

A 57-year-old man presented to the internal medicine department with complaints of sudden upper abdominal pain. He denied fever, changes in the color of his urine or stool, nausea, or vomiting. Physical exams revealed no remarkable findings. Laboratory examination showed a white blood cell (WBC) count of $10,600 / \mu \mathrm{L}$, hemoglobin $(\mathrm{Hb})$ of $14.6 \mathrm{~g} / \mathrm{dL}$, C-reactive protein (CRP) of $2.49 \mathrm{mg} / \mathrm{dL}$, blood urea nitrogen $(B U N)$ of $9.5 \mathrm{mg} / \mathrm{dL}$, serum creatinine (Crea) of $0.63 \mathrm{mg} / \mathrm{dL}$, aspartate aminotransferase concentration of $25 \mathrm{U} / \mathrm{L}$, alanine aminotransferase concentration of $19 \mathrm{U} / \mathrm{L}$, lactate dehydrogenase concentration of $241 \mathrm{U} / \mathrm{L}$, alkaline phosphatase (ALP) concentration of $338 \mathrm{U} / \mathrm{L}, \gamma$-glutamyl transpeptidase ( $\gamma$-GTP) concentration of $66 \mathrm{U} / \mathrm{L}$, serum total protein concentration of $6.65 \mathrm{~g} / \mathrm{dL}$, and serum albumin concentration of $3.59 \mathrm{~g} / \mathrm{dL}$. His serum level of AFP was elevated to $588.9 \mathrm{ng} / \mathrm{mL}$, whereas his carbohydrate antigen 19-9 (CA19-9) and carcinoembryonic antigen (CEA) levels were within the normal ranges. An abdominal computed tomography (CT) scan revealed multiple tumors on his liver suggestive of hepatocellular cancer with part of the tumor rupturing imminently (Figure 1). Five days after admission to our hospital, the mass of the liver ruptured. He was transferred to a different hospital and underwent transarterial chemoembolization (TACE) of the rupturing lesion and HAIC with 5-fluorouracil (5FU)/cisplatin (CDDP) to the others. The patient's AFP levels decreased from 588.9 to $291.7 \mathrm{ng} / \mathrm{mL}$ after one cycle of HAIC with $5 F U / C D D P$ (Figures 2 and 3).

A gastrointestinal scope after TACE and HAIC showed a Borrmann type 2 lesion on the pyloric portion of the lesser curvature, which was histologically diagnosed as suspected hepatoid adenocarcinoma (Figure 4).

Immunohistological staining for AFP and Sal-like protein 4 (SALL4), glypican3, and human epidermal growth receptor 2 (HER2) was negative for AFP and positive for SALL4 and Glypican3 (Figure 5). A liver biopsy confirmed

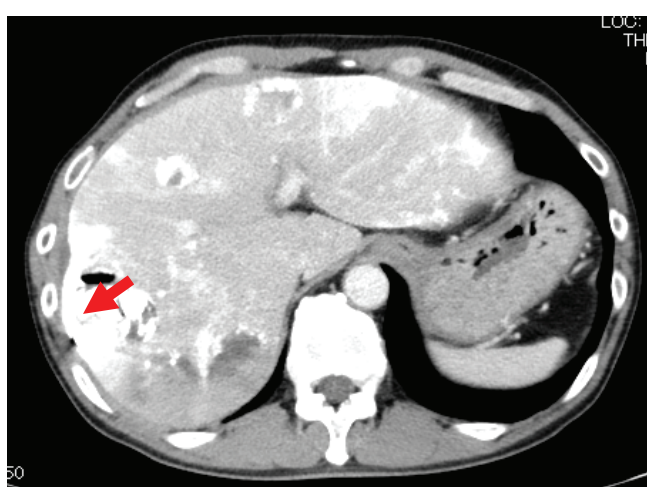

Figure 2: CT scan showing the liver after TACE and HAIC. The red arrow indicates the site of the ruptured mass.

that the lesion was a liver metastasis. Analysis of the liver biopsy specimen revealed that the liver mass was metastatic carcinoma of the liver. Immunohistological staining showed that the specimen was positive for SALL4 and heterochromatin protein 1 (HP1) (Figure 6). Based on these results, the patient was diagnosed with AFPGC and multiple liver metastasis.

He underwent four cycles of systemic chemotherapy with capecitabine (cape)/cisplatin (CDDP) (cape $2000 \mathrm{mg} /$ sqm $>>3,000 \mathrm{mg} /$ body days 1-14, CDDP $80 \mathrm{mg} /$ sqm $>>130 \mathrm{mg} /$ body day 1 , every 3 weeks), which resulted in progressive disease. His AFP levels increased from 297.1 to $4320 \mathrm{ng} / \mathrm{mL}$, and a CT scan revealed progression of the liver metastasis (Figure 3). As the lesion of the liver on S4 was at a high risk of rupturing and HAIC was effective in the previous treatment, we decided to perform TACE and HAIC to the liver metastasis again. Bleeding developed from the gastric lesion after a cycle of HAIC with 5-FU/ CDDP, and the patient underwent a distal gastrectomy and $\mathrm{D} 2+\alpha$ lymph node resection. One month after the gastrectomy, eight cycles of systemic chemotherapy comprising PTX and RAM were administered. As a result, the patient's AFP levels decreased to $2.9 \mathrm{ng} / \mathrm{mL}$ and a CT scan showed that the tumor had vanished (Figure 7). After a three-month drug holiday, chemotherapy with RAM as a maintenance therapy was resumed (Figure 7). The patient has continued with RAM and has so far survived for 19 months after recurrence and is alive without a recurrence.

\section{Discussion}

AFPGC comprises $1.1-8.8 \%$ of all gastric cancers and is a highly aggressive type of gastric cancer compared to non-AFPGC [1-3]. Liver metastasis is frequently observed simultaneously, and metachrony occurs because of the biological characteristics that tend to cause vascular invasion [4-6].

AFPGC has been classified into four histological subtypes: hepatoid, yolk sac tumor, enteroblastic, and common adenocarcinoma type. Positive AFP staining is observed in $54 \%$ and $30 \%$ of hepatoid and yolk sac tumors, respectively. In recent reports, glypican3 and SALL4 staining in addition 


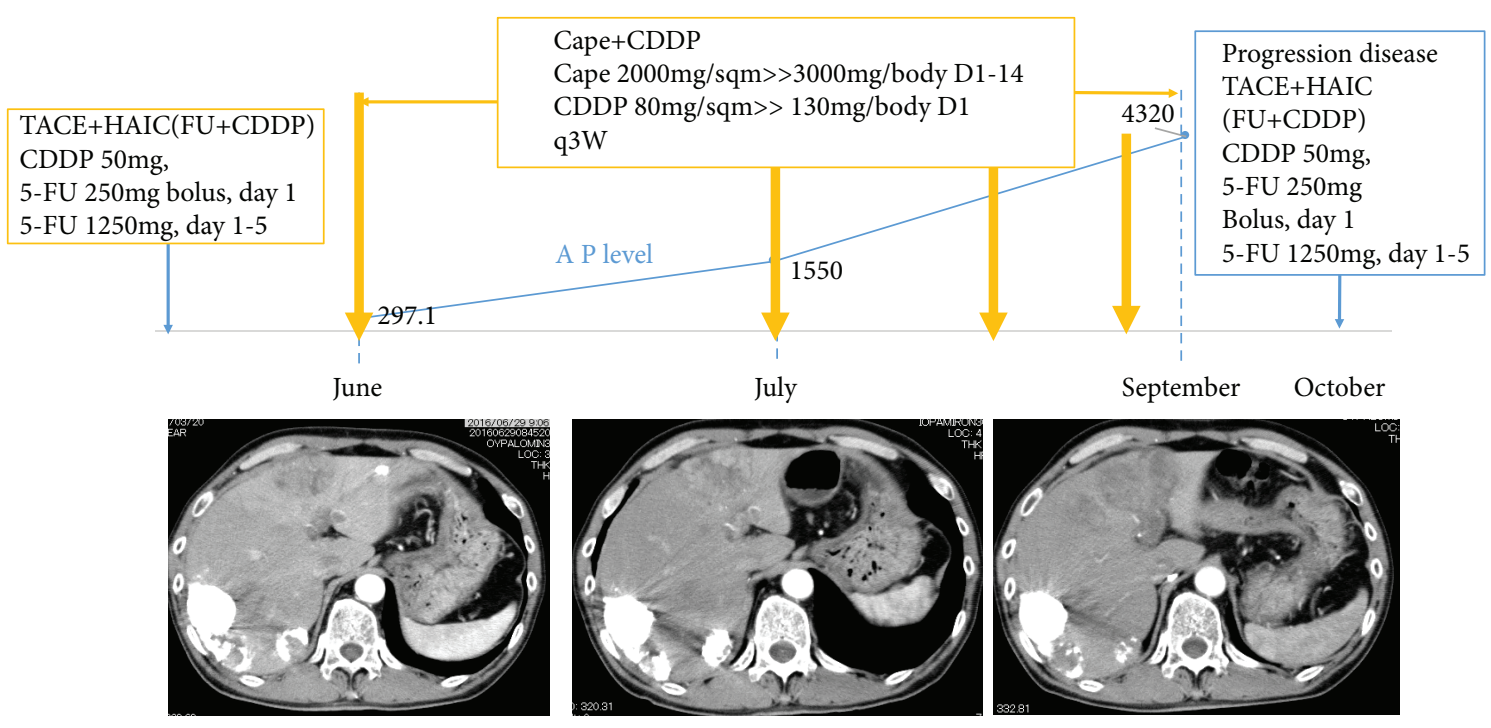

FIGURE 3: Timeline of patient course and treatment. The blue line indicates the AFP level. The patient received four cycles of chemotherapy with cape/CDDP. His AFP level increased from 297.1 to $4320 \mathrm{ng} / \mathrm{mL}$. CT scans revealed the growth of liver metastases.

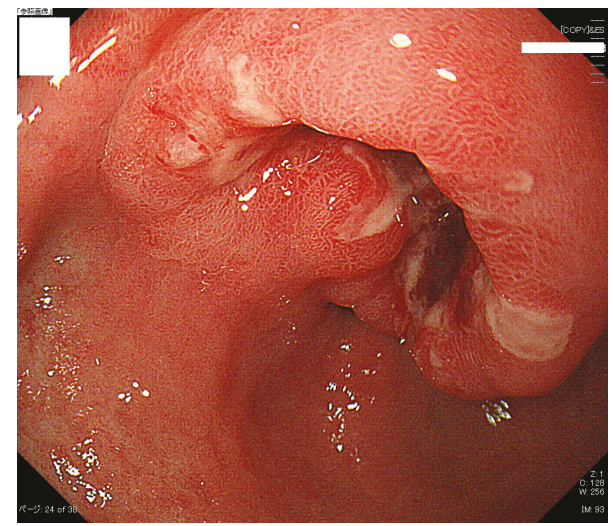

(a)

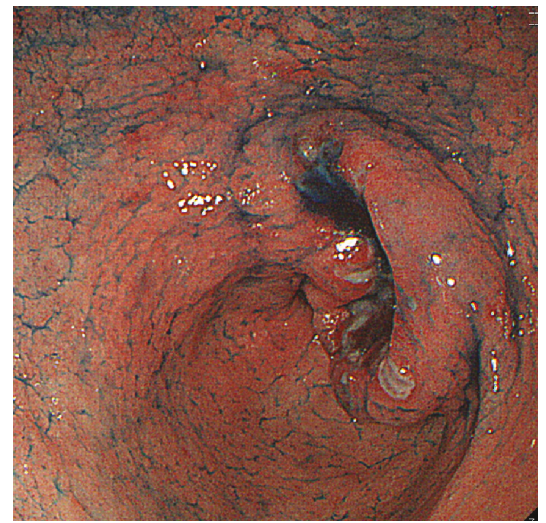

(b)

FIGURE 4: Gastrointestinal scope showing the Borrmann type 2 lesion on the pyloric portion of the lesser curvature that was histologically diagnosed as suspected hepatoid adenocarcinoma.

to AFP protein measurement are effective for the diagnosis of AFPGC. Ushiku and Fukayama reported that, while the AFP-positive staining rate was low (16\% in 32 cases of gastric cancer), the rates for glypican3 and SALL4 were $56 \%$ and $78 \%$, respectively [7]. Although AFP is negative by staining, our case was diagnosed as AFPGC due to findings suggestive of hepatoid adenocarcinoma, SALL4- and glypican3-positive staining, and high serum AFP level.

HAIC is considered effective for the treatment of the liver metastasis of AFPGC. Local intra-arterial chemotherapy such as HAIC has a lower frequency of side effects than that of systemic chemotherapy. Additionally, HAIC is effective because high concentrations of the drug can be injected locally. Sato et al. reported that the combination of HAIC with 5-FU/epirubicin and systemic chemotherapy with S-1 for his patient with AFPGC was effective and safe [8]. There are 17 reports in Japan from 1996 to 2016. Among them were three cases in which HAIC with 5-FU/CDDP was performed. The patients experienced partial response (PR) or complete remission (CR) and have obtained long-term survivals of two years or more after relapse [9-11] (Table 1).

This case was diagnosed as AFPGC with synchronous multiple liver metastases. For liver metastasis rupture during initial treatment, TACE and HAIC with 5FU/ CDDP were performed for volume control. Systemic chemotherapy with cape/CDDP was administered for four cycles. The disease was less responsive to cape/CDDP systemic chemotherapy and developed to progressive disease (PD). As HAIC with 5-FU/CDDP and TACE was initially effective for the liver metastasis in this case, it was repeated. HAIC and TACE effectively controlled the liver metastasis in this case.

AFPGC is very rich in angiogenesis compared to other gastric cancers [12]. Most cancer cells produce vascular endothelial growth factor (VEGF), which is involved in the mitosis of endothelial cells in vitro and is a factor of angiogenesis in vivo $[13,14]$. VEGF-C is an isoform of VEGF that 


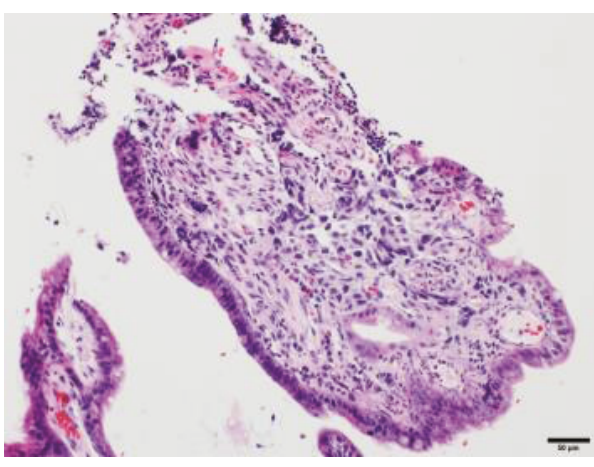

(a)

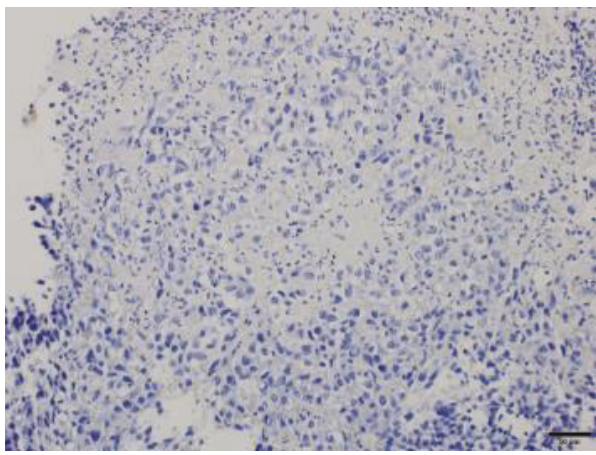

(c)

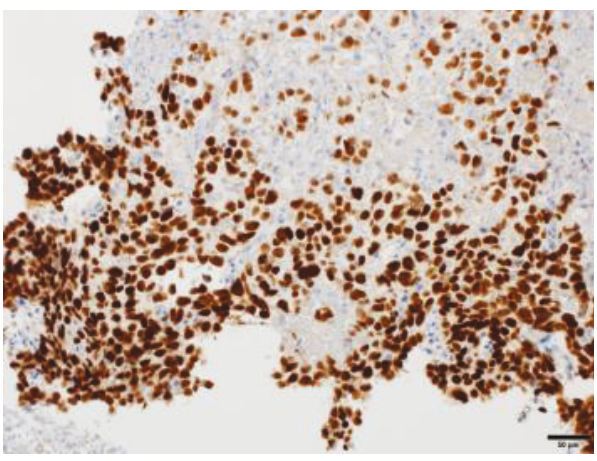

(b)

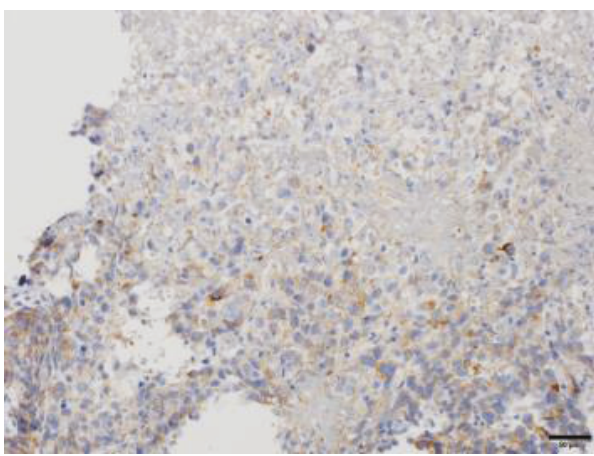

(d)

Figure 5: Gastric biopsy. (a) Hematoxylin and eosin staining indicating suspected hepatoid adenocarcinoma. (b) Positive staining for SALL4. (c) Negative staining for AFP. (d) Focally positive staining for glypican3.

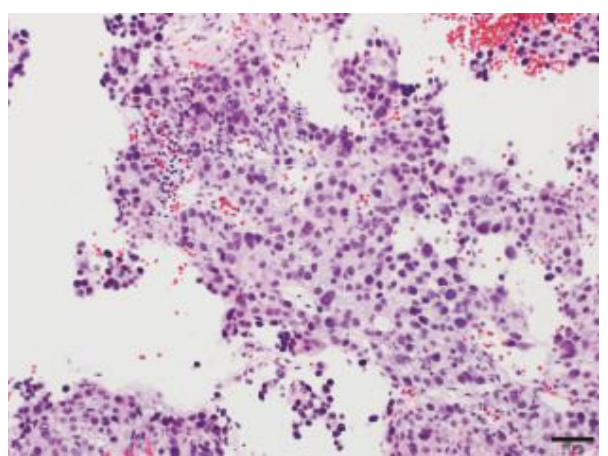

(a)

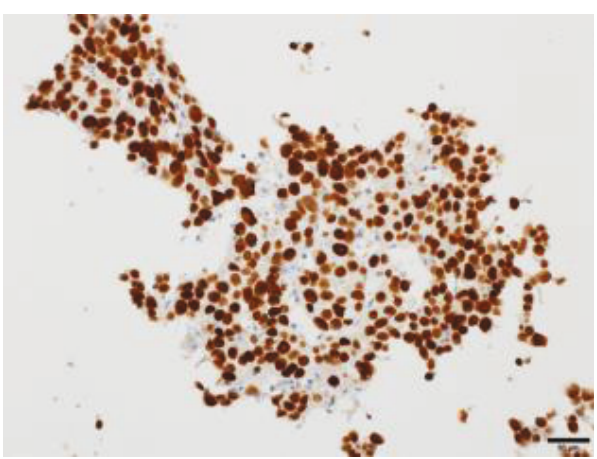

(b)



(c)

Figure 6: Hepatic biopsy. (a) Hematoxylin and eosin staining indicating that the lesion is a liver metastasis. (b) Positive staining for SALL4. (c) Focally positive staining for heterochromatin protein 1 (HP1). 


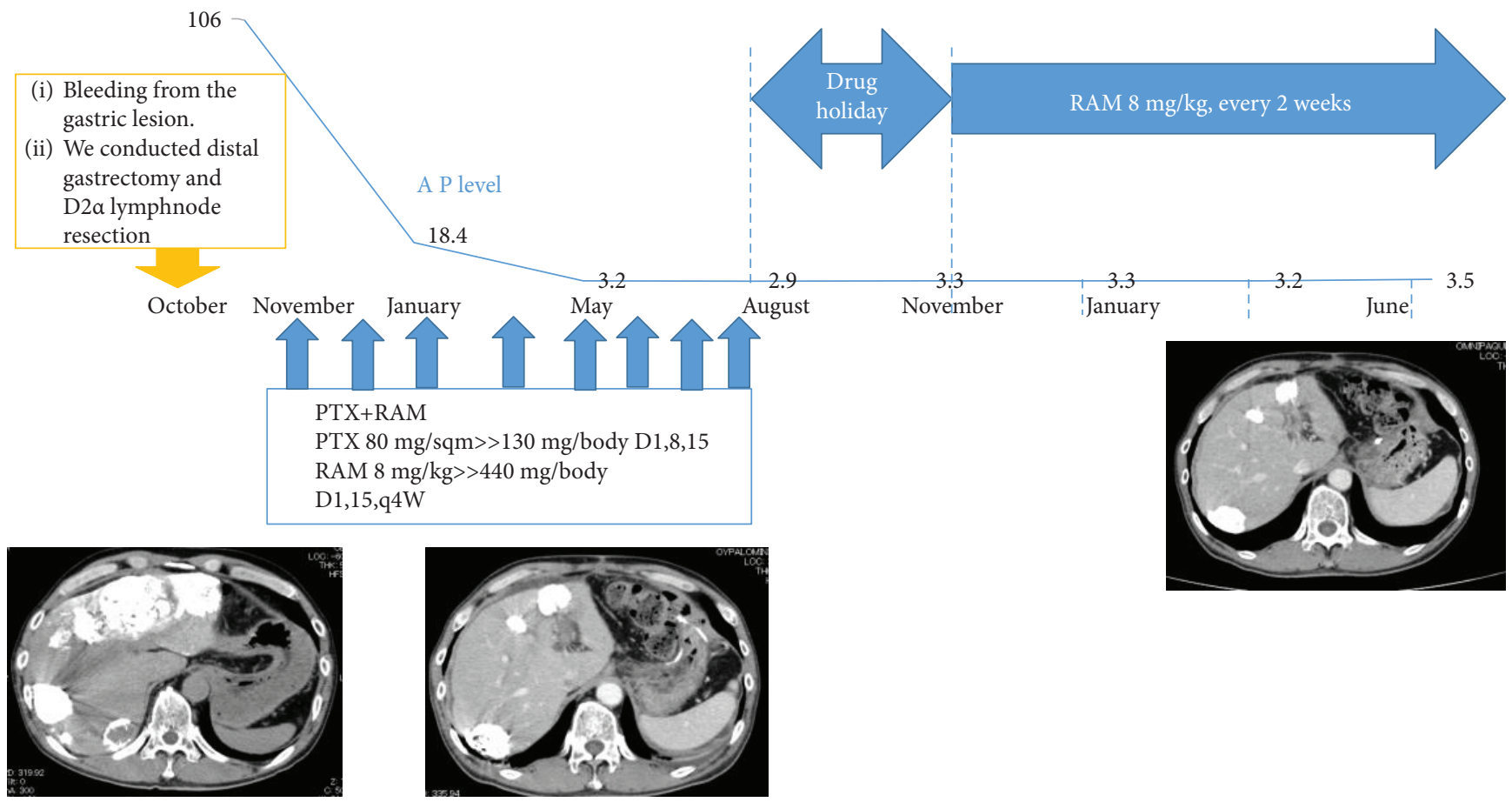

FIGURE 7: After surgery, the patient received eight cycles of chemotherapy comprising PTX and RAM. His AFP levels decreased within the normal range and the lesion in the liver disappeared on a CT scan. After a three-month drug holiday, RAM monotherapy was initiated.

TABLE 1: In Japan, from 1996 to 2016, there are four cases reported in which HAIC with 5FU/CDDP was performed. All of them are a good prognosis.

\begin{tabular}{|c|c|c|c|c|c|c|}
\hline Author (year) & Age & Sex & Rejimen of systemic chemotherapy & Other therapies & Effect & Prognosis \\
\hline $\begin{array}{l}\text { Ozaki et al. [9] } \\
\text { (2008) }\end{array}$ & 73 & Male & - & $\begin{array}{c}\text { Gastrectomy + D3 resection } \\
\text { Partial hepatectomy }\end{array}$ & PR & Unknown \\
\hline $\begin{array}{l}\text { Takada et al. [10] } \\
(2008)\end{array}$ & 75 & Female & $\mathrm{DTX} \rightarrow \mathrm{PTX} \rightarrow \mathrm{CPT}-11$ & $\begin{array}{c}\text { Gastrectomy } \\
\text { Partial hepatectomy } \\
\text { RFA, TAE, radiation }\end{array}$ & CR & $\begin{array}{l}42 \text { months } \\
\text { progression free }\end{array}$ \\
\hline $\begin{array}{l}\text { Tanizaki et al. [11] } \\
\text { (2009) }\end{array}$ & 65 & Female & S-1 & Distal gastrectomy & CR & $\begin{array}{l}28 \text { months } \\
\text { progression free }\end{array}$ \\
\hline Our case (2016) & 57 & Male & $\begin{array}{c}\text { Cape/CDDP } \rightarrow \text { PTX/ } \\
\text { ramucirumab } \rightarrow \text { ramucirumab }\end{array}$ & $\begin{array}{c}\text { TACE } \\
\begin{array}{l}\text { Distal gastrectomy }+D 2 \alpha \\
\text { resection }\end{array}\end{array}$ & CR & $\begin{array}{l}30 \text { months } \\
\text { progression free }\end{array}$ \\
\hline
\end{tabular}

acts as a factor in lymphangiogenesis and promotes lymph ductogenesis. According to Kamei et al., VEGF is highly expressed in AFP-producing gastric cancer compared to the expression in non-AFP-producing gastric cancer [15]. The vascular-rich nature of the liver and lymph node metastases is likely related to VEGF or VEGF-C. RAM inhibits VEGFA, VEGF-C, and VEGF-D by blocking VEGF-R2, and the same targeted drug may be effective for AFP-producing gastric cancer. In the present case, RAM was very effective, leading to a CR after eight cycles of PTX/RAM as adjuvant chemotherapy. This case is the second in Japan to report the effectiveness of RAM.

\section{Conclusion}

Our experience suggested that HAIC with 5-FU/CDDP and systemic chemotherapy with a regimen including RAM may be an effective treatment for AFPGC. HAIC may be effective for the local control of liver metastasis, while RAM may be effective for suppressing progression in the whole body.

\section{Conflicts of Interest}

The authors declare that they have no conflicts of interest.

\section{Acknowledgments}

We would like to thank Editage (http://www.editage.jp) for English language editing.

\section{References}

[1] K. Kono, H. Amemiya, T. Sekikawa et al., "Clinicopathologic features of gastric cancers producing alpha-fetoprotein," Digestive Surgery, vol. 19, no. 5, pp. 359-365, 2002. 
[2] M. Inoue, T. Sano, A. Kuchiba, H. Taniguchi, T. Fukagawa, and H. Katai, "Long-term results of gastrectomy for alphafetoprotein-producing gastric cancer," The British Journal of Surgery, vol. 97, no. 7, pp. 1056-1061, 2010.

[3] X. D. Li, C. P. Wu, M. Ji et al., "Characteristic analysis of $\alpha$ fetoprotein-producing gastric carcinoma in China," World Journal of Surgical Oncology, vol. 11, no. 1, p. 246, 2013.

[4] K. R. McIntire, T. A. Waldmann, C. G. Moertel, and V. L. Go, "Serum alpha-fetoprotein in patients with neoplasms of the gastrointestinal tract," Cancer Research, vol. 35, no. 4, pp. 991-996, 1975.

[5] Y. C. Chang, N. Nagasue, S. Abe et al., "The characters of AFPproducing early gastric cancer," Nihon Geka Gakkai Zasshi, vol. 91, no. 10, pp. 1574-1580, 1990.

[6] T. Motoyama, K. Aizawa, H. Watanabe, M. Fukase, and K. Saito, "Alpha-fetoprotein producing gastric carcinomas: a comparative study of three different subtypes," Acta Pathologica Japonica, vol. 43, no. 11, pp. 654-661, 1993.

[7] T. Ushiku and M. Fukayama, "Fetal type gastric carcinoma and its markers, SALL4, glypican 3 and AFP," Stomach and Intestine(Tokyo), vol. 45, pp. 1950-1958, 2010.

[8] M. Sato, K. Omori, A. Sasaki, M. Nomura, and T. Minamida, "A case of alpha-fetoprotein (AFP)-producing gastric cancer with long-term survival," Nihon Rinsho Geka Gakkai Zasshi (Journal of Japan Surgical Association), vol. 77, no. 2, pp. 346-350, 2016.

[9] R. Ozaki, K. Watanabe, K. Migita et al., "Alpha-fetoproteinproducing hepatoid adenocarcinoma of stomach with liver metastasis responded hepatic arterial infusion chemotherapy. A case report," med. J. Pref, vol. 12, pp. 81-84, 2008.

[10] J. Takada, S. Kenno, T. Aoki, H. Hamada, and Y. Katsuki, “A case in which intra-arterial chemotherapy for simultaneous hepatic metastases markedly improved AFP-producing gastric cancer," Gan to Kagaku Ryoho: Cancer \& Chemotherapy, vol. 36, no. 12, pp. 2326-2329, 2009.

[11] K. Tanizaki, K. Nishioka, K. Kobayashi et al., "A case of AFP producing gastric cancer for which radiation therapy was effective in paraaortic lymph node recurrence," Gan to Kagaku Ryoho: Cancer \& Chemotherapy, vol. 35, no. 12, pp. 20482050, 2008.

[12] N. Koide, A. Nishio, J. Igarashi, S. Kajikawa, W. Adachi, and J. Amano, "Alpha-fetoprotein-producing gastric cancer: histochemical analysis of cell proliferation, apoptosis and angiogenesis," The American Journal of Gastroenterology, vol. 94, no. 6, pp. 1658-1663, 1999.

[13] D. Leung, G. Cachianes, W. Kuang, D. Goeddel, and N. Ferrara, "Vascular endothelial growth factor is a secreted angiogenic mitogen," Science, vol. 246, no. 4935, pp. 13061309, 1989.

[14] D. T. Connolly, D. M. Heuvelman, R. Nelson et al., “Tumor vascular permeability factor stimulates endothelial cell growth and angiogenesis," The Journal of Clinical Investigation, vol. 84, no. 5, pp. 1470-1478, 1989.

[15] S. Kamei, K. Kono, H. Amemiya et al., "Evaluation of VEGF and VEGF-C expression in gastric cancer cells producing $\alpha$-fetoprotein," Journal of Gastroenterology, vol. 38, no. 6 , pp. $540-547,2003$. 


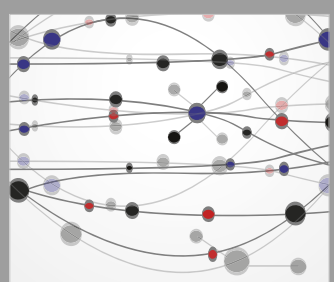

The Scientific World Journal
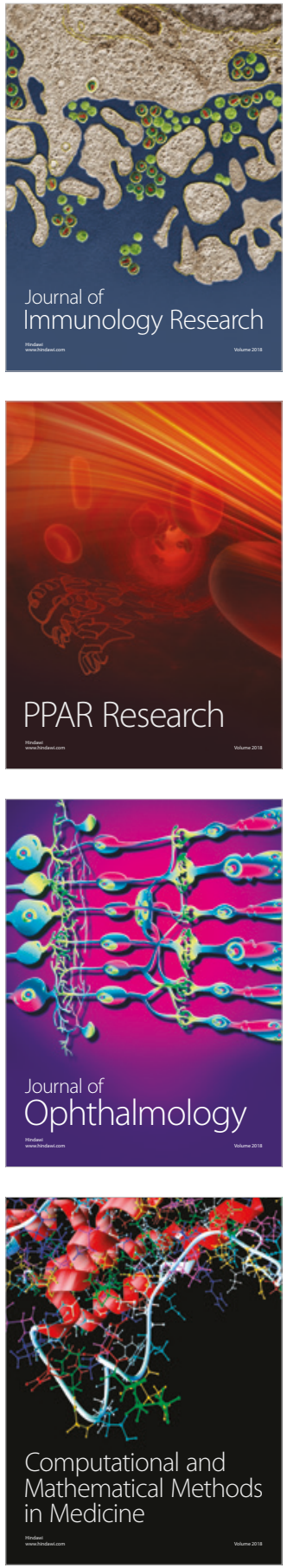

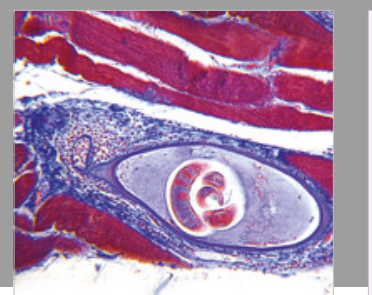

Gastroenterology Research and Practice



\section{Hindawi}

Submit your manuscripts at

www.hindawi.com
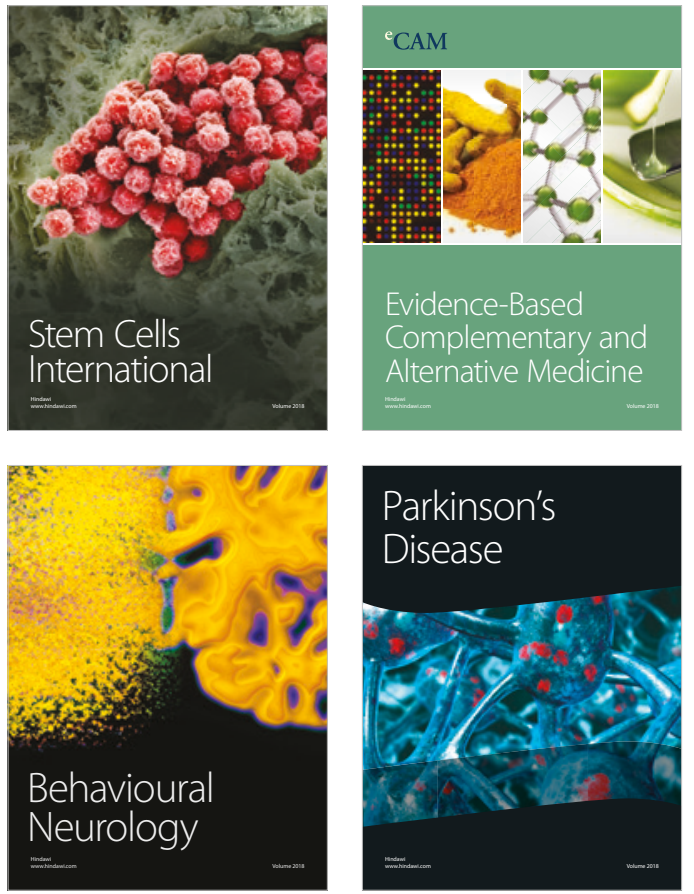

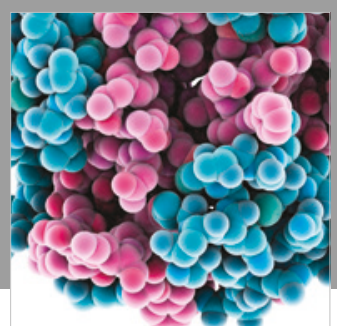

ournal of

Diabetes Research

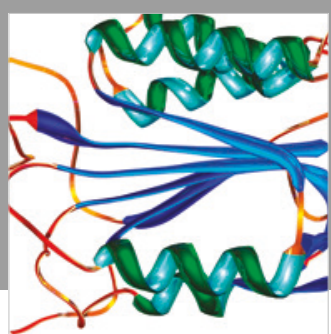

Disease Markers
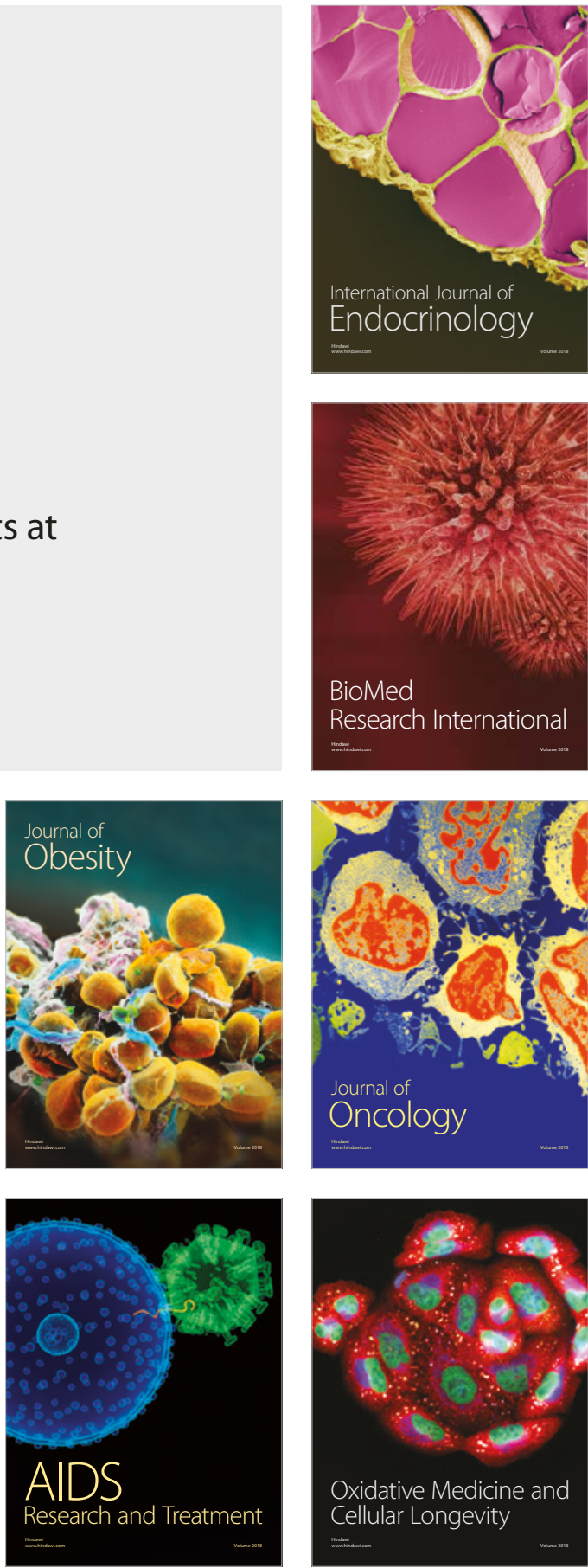